\title{
Delay-dependent robust stability of stochastic delay systems with Markovian switching
}

\author{
Lirong Huang ${ }^{1}$, Xuerong Mao ${ }^{1}$, Feiqi Deng ${ }^{2}$, \\ (1. Department of Statistics and Modelling Science, University of Strathclyde, Glasgow G1 1XH, UK; \\ 2.College of Automation Science and Engineering, South China University of Technology, Guangzhou 510641, China)
}

\begin{abstract}
In recent years, stability of hybrid stochastic delay systems, one of the important issues in the study of stochastic systems, has received considerable attention. However, the existing results do not deal with the structure of the diffusion but estimate its upper bound, which induces conservatism. This paper studies delay-dependent robust stability of hybrid stochastic delay systems. A delay-dependent criterion for robust exponential stability of hybrid stochastic delay systems is presented in terms of linear matrix inequalities (LMIs), which exploits the structure of the diffusion. Numerical examples are given to verify the effectiveness and less conservativeness of the proposed method.
\end{abstract}

Keywords: stochastic systems, time delay, Markovian switching, delay-dependent stability.

\section{Introduction}

Hybrid systems driven by continuous-time Markov chains are used to model various practical systems where they may experience abrupt changes in their structure and parameters, such as failure prone manufacturing systems, power systems and economic systems. An important class of these hybrid systems is the jump linear systems

$$
\dot{x}(t)=A(r(t)) x(t)
$$

where one part of the state $x(t)$ takes value continuously in $R^{n}$ while another part of the state $r(t)$ is the switching signal takeing values in a finite set $S=\{1,2, \cdots, N\}$. Over the past decades, a great deal of attention has been devoted the study of this class of systems (see, e.g., [10], [2], [11] and [15]). Since time delays and uncertainties often appear in practical systems and may induce poor performance and instability, uncertain jump linear systems with time delays have also been studied by many authors (see [10]- [17]). Furthermore, if $r(t)$ is a Markovian chain and environmental noise is taken into account as well as time delay and uncertainty, the system becomes a stochastic delay system with Markovian switching (1), also called a hybrid stochastic delay system (see, e.g., [7]).

Recently, analysis of stability of the Stochastic systems have received much attention (see [4]- [9], [16] and [17]). These works can be classified into two categories according to their dependence on the information about the size of time delays of the system, say, they are either delayindependent results ( [6], [8], [9] and [16]) or delaydependent criteria ( [5], [7], [9] and [17]). Generally, for the cases of small delays, delay-independent results are more conservative than those dependent on the size of delays. However, the existing delay-dependent results do not deal with the structure of the diffusion but estimate its upper bound, which may induce conservativeness.

This paper is concerned with the problem of delaydependent stability of uncertain stochastic delay systems with Markovian switching. By the LMI approach, we present a delay-dependent criterion for exponential stability of uncertain hybrid stochastic delay systems, which exploits the advantages of structure of the diffusion and reduces the conservatism of the existing methods. Numerical examples are conducted to show that our results are considerably less conservative than the existing ones.

\section{Problem statement}

Throughout the paper, unless otherwise specified, we will employ the following notation. Let $\left(\Omega, \mathcal{F},\left\{\mathcal{F}_{t}\right\}_{t \geq 0}, \mathbb{P}\right)$ be a probability space with a natural filtration $\left\{\mathcal{F}_{t}\right\}_{t \geq 0}$ and $\mathbb{E}[\cdot]$ be the expectation operator with respect to the probability measure. If $A$ is a vector or matrix, its transpose is denoted by $A^{T}$. If $P$ is a square matrix, $P>0(P<0)$ means that $P$ is a symmetric positive (negative) definite matrix of appropriate dimensions. $I$ stands for the identity matrix of appropriate dimensions. Denote by $\lambda_{M}(\cdot)$ and $\lambda_{m}(\cdot)$ the maximum and minimum eigenvalue of a matrix respectively. Let $|\cdot|$ denote the Euclidean norm of a vector and its induced norm of a matrix. Unless explicitly stated, matrices are assumed to have real entries and compatible dimensions. Let $h>0$ and $C\left([-h, 0] ; R^{n}\right)$ denote the family of all continuous $R^{n}$-valued functions $\varphi$ on $[-h, 0]$ with the norm $\|\varphi\|=$ $\sup \{|\varphi(\theta)|:-h \leq \theta \leq 0\}$. Let $C_{\mathcal{F}_{0}}^{b}\left([-h, 0] ; R^{n}\right)$ be the family of all $\mathcal{F}_{0}$-measurable bounded $C\left([-h, 0] ; R^{n}\right)$ valued random variables $\xi=\{\xi(\theta):-h \leq \theta \leq 0\}$. Moreover, let $w(t)$ be a scalar Brownian motion defined on the probability space and let $r(t)$ be a right-continuous Markov chain independent of $w(t)$ and taking values in $S=\{1,2, \cdots, N\}$ with generator $\gamma=\left(\gamma_{i j}\right)_{N \times N}$ given by

$\mathbb{P}\{r(t+\Delta)=j \mid r(t)=i\}= \begin{cases}\gamma_{i j} \Delta+o(\Delta), & \text { if } i \neq j \\ 1+\gamma_{i i} \Delta+o(\Delta), & \text { if } i=j\end{cases}$

where $\Delta>0$ and $\gamma_{i j}$ is the transition rate from $i$ to $j$ if $i \neq j$ while $\gamma_{i i}=-\sum_{j \neq i} \gamma_{i j}$.

Let us consider an $n$-dimensional uncertain stochastic de- 
lay system with Markovian switching

$$
\begin{array}{r}
\mathrm{d} x(t)=[A(r(t), t) x(t)+B(r(t), t) x(t-h)] \mathrm{d} t \\
\quad+[C(r(t), t) x(t)+D(r(t), t) x(t-h)] \mathrm{d} w(t)
\end{array}
$$

on $t \geq 0$ with initial data $x_{0}=\{x(\theta):-h \leq \theta \leq 0\}=\xi \in$ $C_{\mathcal{F}_{0}}^{b}\left([-h, 0] ; R^{n}\right)$, where $x(t) \in R^{n}$ is the state vector; positive scalar constant $h$ is time delay of the system; $A(i, t)$, $B(i, t), C(i, t)$ and $D(i, t), i \in S$, are matrix functions with time-varying uncertainties described as follows

$$
\begin{aligned}
& A(i, t)=A_{i}+\Delta A_{i}(t), B(i, t)=B_{i}+\Delta B_{i}(t), \\
& C(i, t)=C_{i}+\Delta C_{i}(t), D(i, t)=D_{i}+\Delta D_{i}(t)
\end{aligned}
$$

where $A_{i}, B_{i}, C_{i}$ and $D_{i}$ are known constant matrices while uncertainties $\Delta A_{i}(t), \Delta B_{i}(t), \Delta C_{i}(t)$ and $\Delta D_{i}(t)$ are assumed to be norm bounded, i.e.,

$$
\begin{aligned}
& \Delta A_{i}(t)=L_{A i} F_{A i}(t) E_{A i}, F_{A i}^{T}(t) F_{A i}(t) \leq I, \\
& \Delta B_{i}(t)=L_{B i} F_{B i}(t) E_{B i}, F_{B i}^{T}(t) F_{B i}(t) \leq I, \\
& \Delta C_{i}(t)=L_{C i} F_{C i}(t) E_{C i}, F_{C i}^{T}(t) F_{C i}(t) \leq I, \\
& \Delta D_{i}(t)=L_{D i} F_{D i}(t) E_{D i}, F_{D i}^{T}(t) F_{D i}(t) \leq I,
\end{aligned}
$$

with known constant matrices $L_{A i}, E_{A i}, L_{B i}, E_{B i}, L_{C i}$, $E_{C i}, L_{D i}$, and $E_{D i}$, and unknown matrix functions $F_{A i}(t)$, $F_{B i}(t), F_{C i}(t)$ and $F_{D i}(t)$ having Lebesgue measurable elements. The parameter uncertainties $\Delta A_{i}(t), \Delta B_{i}(t)$, $\Delta C_{i}(t)$ and $\Delta D_{i}(t), i \in S$, are said to be admissible if conditions (3) hold.

Denote

$$
\begin{aligned}
f(r(t), t) & =A(r(t), t) x(t)+B(r(t), t) x(t-h), \\
g(r(t), t) & =C(r(t), t) x(t)+D(r(t), t) x(t-h)
\end{aligned}
$$

for all $t \geq 0$, which are also written as $f(t)$ and $g(t)$ in short respectively where there is no ambiguity. One can observe that

$$
|f(i, t)|+|g(i, t)| \leq C_{L}|| x_{t}||, \quad \forall(i, t) \in S \times R_{+}
$$

where $x_{t}=\{x(t+\theta):-h \leq \theta \leq 0\}$ and $C_{L}=$ $\max _{1 \leq i \leq N}\left(\left|A_{i}\right|+\left|L_{A i}\right|\left|E_{A i}\right|+\left|B_{i}\right|+\left|\bar{L}_{B i}\right|\left|E_{B i}\right|+\left|C_{i}\right|+\right.$ $\left.\left|L_{C i}\right|\left|E_{C i}\right|+\left|D_{i}\right|+\left|L_{D i}\right|\left|E_{D i}\right|\right)$. This implies that both $f(t)$ and $g(t)$ satisfy the local Lipschitz condition and the linear growth condition. According to Theorem 8.3, p303, [9], there exists a unique continuous solution denoted by $x(t ; \xi)$ or simply $x(t)$ to equation (1). It is known (see, e.g., [9]) that $\left\{x_{t}, r(t)\right\}_{t>0}$ is a $C\left([-h, 0] ; R^{n}\right) \times S \times R_{+}$valued Markov process. Its infinitesimal operator $\mathcal{L}$, acting on functional $V: C\left([-h, 0] ; R^{n}\right) \times S \times R_{+} \rightarrow R$, is defined as, e.g., (3.2) in [7].

In this paper, we are to establish new delay-dependent sufficient conditions for robust exponential stability of uncertain stochastic delay system with Markovian switching (1). For simplicity only, we take single delay $h$ in our model. The proposed method can be easily extended to those cases with multiple and distributed delays.

By the end of this section, let us introduce the following definition and lemmas that are useful for the development of our results.
Definition 1 ( [3]) The uncertain stochastic delay system (1) is said to be robustly exponentially stable in mean square if there is a positive constant $\lambda$ such that

$$
\limsup _{t \rightarrow \infty} \frac{1}{t} \log \mathbb{E}|x(t ; \xi)|^{2} \leq-\lambda
$$

for all admissible uncertainties (3).

Lemma 1 ( [13]) For any constant matrix $M \in R^{q \times l}$, inequality

$2 u^{T} M v \leq r u^{T} M G M^{T} u+\frac{1}{r} v^{T} G^{-1} v, \quad u \in R^{q}, v \in R^{l}$

holds for any pair of symmetric positive definite matrix $G \in R^{l \times l}$ and positive number $r>0$.

Lemma 2 ( [1]) For any pair of symmetric positive definite constant matrix $G \in R^{l \times l}$ and scalar $r>0$, if there exists a vector function $v:[0, r] \rightarrow R^{l}$ such that integrals $\int_{0}^{r} v^{T}(s) G v(s) \mathrm{d} s$ and $\int_{0}^{r} v(s) \mathrm{d} s$ are well defined, then the following inequality holds

$$
r \int_{0}^{r} v^{T}(s) G v(s) \mathrm{d} s \geq\left(\int_{0}^{r} v(s) \mathrm{d} s\right)^{T} G\left(\int_{0}^{r} v(s) \mathrm{d} s\right) .
$$

Lemma 3 ( [7, 17]) Assume that $\phi: C\left([-h, 0] ; R^{n}\right) \times$ $S \times R_{+} \rightarrow R^{n}, i \in S, t \geq h$ and $r(t)=i$. Define

$$
\begin{aligned}
& v_{1}(x(t), i, t)=x^{T}(t) M_{i} x(t), \\
& v_{2}\left(x_{t}, i, t\right)=\int_{-h}^{0} \int_{t+\theta}^{t} \phi^{T}\left(x_{s}, i, s\right) G \phi\left(x_{s}, i, s\right) \mathrm{d} s \mathrm{~d} \theta
\end{aligned}
$$

where $M_{i}$ and $G, i \in S$, are symmetric matrices. Then

$$
\begin{aligned}
\mathcal{L} v_{1}\left(x_{t}, i, t\right)= & 2 x^{T}(t) M_{i} f(i, t)+g^{T}(i, t) M_{i} g(i, t) \\
& +\sum_{j=1}^{N} \gamma_{i j} x^{T}(t) M_{j} x(t), \\
\mathcal{L} v_{2}\left(x_{t}, i, t\right)= & h \phi^{T}\left(x_{t}, i, t\right) G \phi\left(x_{t}, i, t\right) \\
& -\int_{t-h}^{t} \phi^{T}\left(x_{s}, r(s), s\right) G \phi\left(x_{s}, r(s), s\right) \mathrm{d} s .
\end{aligned}
$$

\section{Delay-dependent exponential stability}

Sufficient conditions for robust exponential stability of uncertain hybrid stochastic delay system (1) are proposed as follows.

Theorem 1 The uncertain stochastic delay system with Markovian switching (1) is robustly mean-square exponentially stable provided that there exist matrices $P_{i 11}>0$, $Q>0, R>0, P_{i 21}, P_{i 22}, P_{i 23}, P_{i 31}, P_{i 32}, P_{i 33}$, and scalar numbers $\beta_{A i}>0, \beta_{B i}>0, \beta_{C i}>0$ and $\beta_{D i}>0$ such that 


$$
\left[\begin{array}{ccccccccc}
\Phi_{i 11} & * & * & * & * & * & * & * & * \\
\Phi_{i 21} & \Phi_{i 22} & * & * & * & * & * & * & * \\
\Phi_{i 31} & \Phi_{i 32} & \Phi_{i 33} & * & * & * & * & * & * \\
\Phi_{i 41} & \Phi_{i 42} & \Phi_{i 43} & \Phi_{i 44} & * & 0 & 0 & 0 & 0 \\
\Phi_{i 51} & \Phi_{i 52} & \Phi_{i 53} & \Phi_{i 54} & \Phi_{i 55} & 0 & 0 & 0 & 0 \\
L_{A i}^{T} P_{i 21} & L_{A i}^{T} P_{i 22} & L_{A i}^{T} P_{i 23} & 0 & 0 & -\beta_{A i} I & 0 & 0 & 0 \\
L_{B i}^{T} P_{i 21} & L_{B i}^{T} P_{i 22} & L_{B i}^{T} P_{i 23} & 0 & 0 & 0 & -\beta_{B i} I & 0 & 0 \\
L_{C i}^{T} P_{i 31} & L_{C i}^{T} P_{i 32} & L_{C i}^{T} P_{i 33} & 0 & 0 & 0 & 0 & -\beta_{C i} I & 0 \\
L_{D i}^{T} P_{i 31} & L_{D i}^{T} P_{i 32} & L_{D i}^{T} P_{i 33} & 0 & 0 & 0 & 0 & 0 & -\beta_{D i} I
\end{array}\right]<0
$$

LMI (6) holds for all $i \in S$, where

$$
\begin{aligned}
\Phi_{i 11}= & P_{i 21}^{T}\left(A_{i}+B_{i}\right)+\left(A_{i}+B_{i}\right)^{T} P_{i 21}+P_{i 31}^{T}\left(C_{i}+D_{i}\right) \\
& +\left(C_{i}+D_{i}\right)^{T} P_{i 31}+\sum_{j=1}^{N} \gamma_{i j} P_{j 11}+\beta_{A i} E_{A i}^{T} E_{A i} \\
& +\beta_{B i} E_{B i}^{T} E_{B i}+\beta_{C i} E_{C i}^{T} E_{C i}+\beta_{D i} E_{D i}^{T} E_{D i}, \\
\Phi_{i 21}= & P_{i 22}^{T}\left(A_{i}+B_{i}\right)+P_{i 32}^{T}\left(C_{i}+D_{i}\right)+P_{i 11}-P_{i 21}, \\
\Phi_{i 31}= & P_{i 23}^{T}\left(A_{i}+B_{i}\right)+P_{i 33}^{T}\left(C_{i}+D_{i}\right)-P_{i 31}, \\
\Phi_{i 41}= & h\left(B_{i}^{T} P_{i 21}+D_{i}^{T} P_{i 31}+\beta_{B i} E_{B i}^{T} E_{B i}+\beta_{D i} E_{D i}^{T} E_{D i}\right), \\
\Phi_{i 51}= & \left(B_{i}^{T} P_{i 21}+D_{i}^{T} P_{i 31}+\beta_{B i} E_{B i}^{T} E_{B i}+\beta_{D i} E_{D i}^{T} E_{D i}\right), \\
\Phi_{i 22}= & -P_{i 22}^{T}-P_{i 22}+h Q, \Phi_{i 32}=-P_{i 23}^{T}-P_{i 32}, \\
\Phi_{i 42}= & h\left(B_{i}^{T} P_{i 22}+D_{i}^{T} P_{i 32}\right), \\
\Phi_{i 52}= & \left(B_{i}^{T} P_{i 22}+D_{i}^{T} P_{i 32}\right), \\
\Phi_{i 33}= & -P_{i 33}^{T}-P_{i 33}+P_{i 11}+h R, \\
\Phi_{i 43}= & h\left(B_{i}^{T} P_{i 23}+D_{i}^{T} P_{i 33}\right), \\
\Phi_{i 53}= & \left(B_{i}^{T} P_{i 23}+D_{i}^{T} P_{i 33}\right), \\
\Phi_{i 44}= & -h Q+h^{2}\left(\beta_{B i} E_{B i}^{T} E_{B i}+\beta_{D i} E_{D i}^{T} E_{D i}\right), \\
\Phi_{i 54}= & h\left(\beta_{B i} E_{B i}^{T} E_{B i}+\beta_{D i} E_{D i}^{T} E_{D i}\right), \\
\Phi_{i 55}= & -R+\beta_{B i} E_{B i}^{T} E_{B i}+\beta_{D i} E_{D i}^{T} E_{D i},
\end{aligned}
$$

and entries denoted by $*$ can be readily inferred from symmetry of the matrices.

Proof. By notation (4), we can rewrite the hybrid stochastic delay system (1) in short as

$$
\mathrm{d} x(t)=f(r(t), t) \mathrm{d} t+g(r(t), t) \mathrm{d} w(t)
$$

on $t \geq 0$ with initial data $\xi$.

So we have

$$
x\left(t_{2}\right)-x\left(t_{1}\right)=\int_{t_{1}}^{t_{2}} f(r(s), s) \mathrm{d} s+g(r(s), s) \mathrm{d} w(s)
$$

for all $t_{2} \geq t_{1} \geq 0$.

By (4) and (8), we observe that

$$
\begin{aligned}
f(i, t) & =\left(A_{i}(t)+B_{i}(t)\right) x(t) \\
& -B_{i}(t) \int_{t-h}^{t} f(r(s), s) \mathrm{d} s+g(r(s), s) \mathrm{d} w(s),(9) \\
g(i, t) & =\left(C_{i}(t)+D_{i}(t)\right) x(t) \\
& -D_{i}(t) \int_{t-h}^{t} f(r(s), s) \mathrm{d} s+g(r(s), s) \mathrm{d} w(s)(10)
\end{aligned}
$$

for all $i \in S$ and $t \geq h$.

Choose a Lyapunov-Krasovskii functional candidate for system (7) as follows:

$$
V\left(x_{t}, i, t\right)=V_{1}(x(t), i, t)+V_{2}\left(x_{t}, i, t\right)+V_{3}\left(x_{t}, i, t\right),
$$

where

$$
\begin{aligned}
& V_{1}(x(t), i, t)=x^{T}(t) P_{i 11} x(t), \\
& V_{2}\left(x_{t}, i, t\right)=\int_{-h}^{0} \int_{t+\theta}^{t} f^{T}(r(s), s) Q f(r(s), s) \mathrm{d} s \mathrm{~d} \theta, \\
& V_{3}\left(x_{t}, i, t\right)=\int_{-h}^{0} \int_{t+\theta}^{t} g^{T}(r(s), s) R g(r(s), s) \mathrm{d} s \mathrm{~d} \theta
\end{aligned}
$$

for $i \in S$ and $t \geq h$.

Let $\varepsilon$ be a positive real number to be determined. For any $\tau \geq h$, by the generalized Itô's lemma (see, e.g., [16]) and Fubini's lemma, we have

$$
\begin{aligned}
& \mathbb{E}\left[e^{\varepsilon \tau} V\left(x_{\tau}, r(\tau), \tau\right)-e^{\varepsilon h} V\left(x_{h}, r(h), h\right)\right] \\
& =\mathbb{E} \int_{h}^{\tau} e^{\varepsilon t}\left[\varepsilon V\left(x_{t}, r(t), t\right)+\mathcal{L} V\left(x_{t}, r(t), t\right)\right] \mathrm{d} t \\
& =\int_{h}^{\tau} e^{\varepsilon t} \mathbb{E}\left[\varepsilon V\left(x_{t}, r(t), t\right)+\mathcal{L} V\left(x_{t}, r(t), t\right)\right] \mathrm{d} t,
\end{aligned}
$$

where

$$
\begin{aligned}
\mathcal{L} V\left(x_{t}, r(t), t\right)= & \mathcal{L} V_{1}(x(t), r(t), t)+\mathcal{L} V_{2}\left(x_{t}, r(t), t\right) \\
& +\mathcal{L} V_{3}\left(x_{t}, r(t), t\right) .
\end{aligned}
$$

By Lemma 3, for any $i \in S$, we have

$$
\begin{aligned}
\mathcal{L} V_{1}(x(t), i, t)= & 2 x^{T}(t) P_{i 11} f(i, t)+g^{T}(i, t) P_{i 11} g(i, t) \\
& +\sum_{j=1}^{N} \gamma_{i j} x^{T}(t) P_{j 11} x(t), \\
\mathcal{L} V_{2}\left(x_{t}, i, t\right)= & h f^{T}(i, t) Q f(i, t) \\
& -\int_{t-h}^{t} f^{T}(r(s), s) Q f(r(s), s) \mathrm{d} s,(15) \\
\mathcal{L} V_{3}\left(x_{t}, i, t\right)= & h g^{T}(i, t) R g(i, t) \\
& -\int_{t-h}^{t} g^{T}(r(s), s) R g(r(s), s) \mathrm{d} s .(16)
\end{aligned}
$$

Denote

$y_{i}(t)=\left[\begin{array}{c}x(t) \\ f(i, t) \\ g(i, t)\end{array}\right] \quad$ and $\quad P_{i}=\left[\begin{array}{ccc}P_{i 11} & 0 & 0 \\ P_{i 21} & P_{i 22} & P_{i 23} \\ P_{i 31} & P_{i 32} & P_{i 33}\end{array}\right]$ 
for all $i \in S$. By equalities (9), (10) and (17), we have

$$
\begin{aligned}
& 2 x^{T}(t) P_{i 11} f(i, t) \\
= & y_{i}^{T}(t)\left(P_{i}^{T} G_{i}+G_{i}^{T} P_{i}\right) y_{i}(t)+2 y_{i}^{T}(t) P_{i}^{T} \Delta G_{i} x(t) \\
& -2 y_{i}^{T}(t) P_{i}^{T}\left[\begin{array}{ll}
0 & B_{i}^{T} D_{i}^{T}
\end{array}\right]^{T} \\
& \cdot \int_{t-h}^{t} f(r(s), s) \mathrm{d} s+g(r(s), s) \mathrm{d} w(s) \\
& -2 y_{i}^{T}(t) P_{i}^{T}\left[0 \Delta B_{i}^{T}(t) \Delta D_{i}^{T}(t)\right]^{T} \\
& \cdot \int_{t-h}^{t} f(r(s), s) \mathrm{d} s+g(r(s), s) \mathrm{d} w(s)
\end{aligned}
$$

where $G_{i}=\left[\begin{array}{ccc}0 & I & 0 \\ A_{i}+B_{i}-I & 0 \\ C_{i}+D_{i} & 0 & -I\end{array}\right]$ and $\Delta G_{i}=\Delta \bar{A}_{i}+$ $\Delta \bar{B}_{i}+\Delta \bar{C}_{i}+\Delta \bar{D}_{i}$ with $\Delta \bar{A}_{i}=\left[0 \Delta A_{i}^{T}(t) 0\right]^{T}, \Delta \bar{B}_{i}=$ $\left[\begin{array}{lll}0 & \Delta B_{i}^{T}(t) & 0\end{array}\right]^{T}, \Delta \bar{C}_{i}=\left[\begin{array}{lll}0 & 0 & \Delta C_{i}^{T}(t)\end{array}\right]^{T}$ and $\Delta \bar{D}_{i}=$

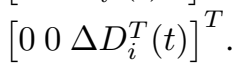

For any $i \in S$, let $z_{i}^{T}(t)=\left[z_{i 1}^{T}(t) z_{i 2}^{T}(t) z_{i 3}^{T}(t) z_{i 4}^{T}(t)\right.$ $\left.z_{i 5}^{T}(t)\right]^{T}=\left[x^{T}(t) f^{T}(i, t) g^{T}(i, t) \frac{-1}{h} \int_{t-h}^{t} f^{T}(r(s), s) \mathrm{d} s\right.$ $\left.-\int_{t-h}^{t} g^{T}(r(s), s) \mathrm{d} w(s)\right]^{T}$ and let $\tilde{E}_{A i}=\left[\begin{array}{lllll}E_{A i} & 0 & 0 & 0 & 0\end{array}\right]$, $\tilde{L}_{A i}^{T}=\left[\begin{array}{llllll}L_{A i}^{T} P_{i 21} & L_{A i}^{T} P_{i 22} & L_{A i}^{T} P_{i 23} & 0 & 0\end{array}\right]^{T}, \quad \tilde{E}_{B i}=$ $\left[\begin{array}{lllll}E_{B i} & 0 & 0 & h E_{B i} & E_{B i}\end{array}\right], \tilde{L}_{B i}^{T}=\left[\begin{array}{lllll}L_{B i}^{T} P_{i 21} & L_{B i}^{T} P_{i 22} & L_{B i}^{T} P_{i 23} & 0 & 0\end{array}\right]^{T}$ $\tilde{E}_{C i}=\left[\begin{array}{lllll}E_{C i} & 0 & 0 & 0 & 0\end{array}\right], \tilde{L}_{C i}^{T}=\left[\begin{array}{lllll}L_{C i}^{T} P_{i 31} & L_{C i}^{T} P_{i 32} & L_{C i}^{T} P_{i 33} & 0 & 0\end{array}\right]^{T}$,

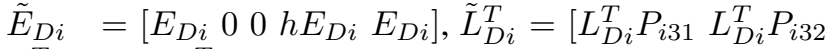
$\left.L_{D i}^{T} P_{i 33} \quad 0 \quad 0\right]^{T}$.

Then, by Lemma 1, we have

$$
\begin{aligned}
& 2 y_{i}^{T}(t) P_{i}^{T} \Delta \bar{A}_{i} x(t)=2 z_{i}^{T}(t) \tilde{L}_{A i} F_{A i}(t) \tilde{E}_{A i} z_{i}(t) \\
& \leq \beta_{A i}^{-1} z_{i}^{T}(t) \tilde{L}_{A i} \tilde{L}_{A i}^{T} z_{i}(t)+\beta_{A i} z_{i}^{T}(t) \tilde{E}_{A i}^{T} \tilde{E}_{A i} z_{i}(t), \\
& 2 y_{i}^{T}(t) P_{i}^{T} \Delta \bar{C}_{i} x(t)=2 z_{i}^{T}(t) \tilde{L}_{C i} F_{C i}(t) \tilde{E}_{C i} z_{i}(t) \\
& \leq \beta_{C i}^{-1} z_{i}^{T}(t) \tilde{L}_{C i} \tilde{L}_{C i}^{T} z_{i}(t)+\beta_{C i} z_{i}^{T}(t) \tilde{E}_{C i}^{T} \tilde{E}_{C i} z_{i}(t),
\end{aligned}
$$

and

$$
\begin{aligned}
& 2 y_{i}^{T}(t) P_{i}^{T} \Delta \bar{B}_{i} \\
& \cdot\left(x(t)-\int_{t-h}^{t} f(r(s), s) \mathrm{d} s+g(r(s), s) \mathrm{d} w(s)\right) \\
& =2 z^{T}(t) \tilde{L}_{B i} F_{B i}(t) \tilde{E}_{B i} z(t) \\
& \leq \beta_{B i}^{-1} z_{i}^{T}(t) \tilde{L}_{B i} \tilde{L}_{B i}^{T} z_{i}(t)+\beta_{B i} z_{i}^{T}(t) \tilde{E}_{B i}^{T} \tilde{E}_{B i} z_{i}(t), \\
& 2 y_{i}^{T}(t) P_{i}^{T} \Delta \bar{D}_{i} \\
& \cdot\left(x(t)-\int_{t-h}^{t} f(r(s), s) \mathrm{d} s+g(r(s), s) \mathrm{d} w(s)\right) \\
& =2 z^{T}(t) \tilde{L}_{D i} F_{D i}(t) \tilde{E}_{D i} z(t) \\
& \leq \beta_{D i}^{-1} z_{i}^{T}(t) \tilde{L}_{D i} \tilde{L}_{D i}^{T} z_{i}(t)+\beta_{D i} z_{i}^{T}(t) \tilde{E}_{D i}^{T} \tilde{E}_{D i} z_{i}(t),
\end{aligned}
$$

which imply

$$
\begin{aligned}
& 2 y_{i}^{T}(t) P_{i}^{T}\left(\Delta G_{i} x(t)-\left[0 \Delta B_{i}^{T}(t) \Delta D_{i}^{T}(t)\right]^{T}\right. \\
& \left.\cdot \int_{t-h}^{t} f(r(s), s) \mathrm{d} s+g(r(s), s) \mathrm{d} w(s)\right) \\
& \leq z_{i}^{T}(t)\left[\beta_{A i}^{-1} \tilde{L}_{A i} \tilde{L}_{A i}^{T}+\beta_{A i} \tilde{E}_{A i}^{T} \tilde{E}_{A i}+\beta_{B i}^{-1} \tilde{L}_{B i} \tilde{L}_{B i}^{T}\right. \\
& +\beta_{B i} \tilde{E}_{B i}^{T} \tilde{E}_{B i}+\beta_{C i}^{-1} \tilde{L}_{C i} \tilde{L}_{C i}^{T}+\beta_{C i} \tilde{E}_{C i}^{T} \tilde{E}_{C i} \\
& \left.+\beta_{D i}^{-1} \tilde{L}_{D i} \tilde{L}_{D i}^{T}+\beta_{D i} \tilde{E}_{D i}^{T} \tilde{E}_{D i}\right] z_{i}(t) .
\end{aligned}
$$

Substitution of (18)-(19) into (14) yields

$$
\mathcal{L} V_{1}(x(t), i, t) \leq z_{i}^{T}(t) \Gamma_{i} z_{i}(t)
$$

where symmetric matrix $\Gamma_{i}$ is given as follows

$$
\Gamma_{i}=\left[\begin{array}{ccccc}
\Gamma_{i 11} & * & * & * & * \\
\Gamma_{i 21} & \Gamma_{i 22} & * & * & * \\
\Gamma_{i 31} & \Gamma_{i 32} & \Gamma_{i 33} & * & * \\
\Gamma_{i 41} & \Gamma_{i 42} & \Gamma_{i 43} & \Gamma_{i 44} & * \\
\Gamma_{i 51} & \Gamma_{i 52} & \Gamma_{i 53} & \Gamma_{i 54} & \Gamma_{i 55}
\end{array}\right]
$$

with

$\Gamma_{i 11}=P_{i 21}^{T}\left(A_{i}+B_{i}\right)+\left(A_{i}+B_{i}\right)^{T} P_{i 21}+P_{i 31}^{T}\left(C_{i}+D_{i}\right)$

$$
+\left(C_{i}+D_{i}\right)^{T} P_{i 31}+\sum_{j=1}^{N} \gamma_{i j} P_{j 11}+\beta_{A i} E_{A i}^{T} E_{A i}
$$$$
+\beta_{B i} E_{B i}^{T} E_{B i}+\beta_{C i} E_{C i}^{T} E_{C i}+\beta_{D i} E_{D i}^{T} E_{D i}
$$$$
+\beta_{A i}^{-1} P_{i 21}^{T} L_{A i} L_{A i}^{T} P_{i 21}+\beta_{B i}^{-1} P_{i 21}^{T} L_{B i} L_{B i}^{T} P_{i 21}
$$$$
+\beta_{C i}^{-1} P_{i 31}^{T} L_{C i} L_{C i}^{T} P_{i 31}+\beta_{D i}^{-1} P_{i 31}^{T} L_{D i} L_{D i}^{T} P_{i 31},
$$$$
\Gamma_{i 21}=P_{i 22}^{T}\left(A_{i}+B_{i}\right)+P_{i 32}^{T}\left(C_{i}+D_{i}\right)+P_{i 11}-P_{i 21}
$$$$
+\beta_{A i}^{-1} P_{i 22}^{T} L_{A i} L_{A i}^{T} P_{i 21}+\beta_{B i}^{-1} P_{i 22}^{T} L_{B i} L_{B i}^{T} P_{i 21}
$$$$
+\beta_{C i}^{-1} P_{i 32}^{T} L_{C i} L_{C i}^{T} P_{i 31}+\beta_{D i}^{-1} P_{i 32}^{T} L_{D i} L_{D i}^{T} P_{i 31} \text {, }
$$

$\Gamma_{i 31}=P_{i 23}^{T}\left(A_{i}+B_{i}\right)+P_{i 33}^{T}\left(C_{i}+D_{i}\right)-P_{i 31}$

$+\beta_{A i}^{-1} P_{i 23}^{T} L_{A i} L_{A i}^{T} P_{i 21}+\beta_{B i}^{-1} P_{i 23}^{T} L_{B i} L_{B i}^{T} P_{i 21}$

$+\beta_{C i}^{-1} P_{i 33}^{T} L_{C i} L_{C i}^{T} P_{i 31}+\beta_{D i}^{-1} P_{i 33}^{T} L_{D i} L_{D i}^{T} P_{i 31}$,

$\Gamma_{i 41}=h\left(B_{i}^{T} P_{i 21}+D_{i}^{T} P_{i 31}+\beta_{B i} E_{B i}^{T} E_{B i}+\beta_{D i} E_{D i}^{T} E_{D i}\right)$,

$\Gamma_{i 51}=\left(B_{i}^{T} P_{i 21}+D_{i}^{T} P_{i 31}+\beta_{B i} E_{B i}^{T} E_{B i}+\beta_{D i} E_{D i}^{T} E_{D i}\right)$,

$\Gamma_{i 22}=-P_{i 22}^{T}-P_{i 22}+\beta_{A i}^{-1} P_{i 22}^{T} L_{A i} L_{A i}^{T} P_{i 22}$

$+\beta_{B i}^{-1} P_{i 22}^{T} L_{B i} L_{B i}^{T} P_{i 22}+\beta_{C i}^{-1} P_{i 32}^{T} L_{C i} L_{C i}^{T} P_{i 32}$

$+\beta_{D i}^{-1} P_{i 32}^{T} L_{D i} L_{D i}^{T} P_{i 32}$,

$\Gamma_{i 32}=-P_{i 23}^{T}-P_{i 32}+\beta_{A i}^{-1} P_{i 23}^{T} L_{A i} L_{A i}^{T} P_{i 22}$

$+\beta_{B i}^{-1} P_{i 23}^{T} L_{B i} L_{B i}^{T} P_{i 22}+\beta_{C i}^{-1} P_{i 33}^{T} L_{C i} L_{C i}^{T} P_{i 32}$

$+\beta_{D i}^{-1} P_{i 33}^{T} L_{D i} L_{D i}^{T} P_{i 32}$,

$\Gamma_{i 42}=h\left(B_{i}^{T} P_{i 22}+D_{i}^{T} P_{i 32}\right), \Gamma_{52}=\left(B_{i}^{T} P_{i 22}+D_{i}^{T} P_{i 32}\right)$,

$\Gamma_{i 33}=-P_{i 33}^{T}-P_{i 33}+P_{i 11}+\beta_{A i}^{-1} P_{i 23}^{T} L_{A i} L_{A i}^{T} P_{i 23}$

$+\beta_{B i}^{-1} P_{i 23}^{T} L_{B i} L_{B i}^{T} P_{i 23}+\beta_{C i}^{-1} P_{i 33}^{T} L_{C i} L_{C i}^{T} P_{i 33}$

$+\beta_{D i}^{-1} P_{i 33}^{T} L_{D i} L_{D i}^{T} P_{i 33}$,

$\Gamma_{i 43}=h\left(B_{i}^{T} P_{i 23}+D_{i}^{T} P_{i 33}\right), \Gamma_{53}=\left(B_{i}^{T} P_{i 23}+D_{i}^{T} P_{i 33}\right)$,

$\Gamma_{i 44}=h^{2}\left(\beta_{B i} E_{B i}^{T} E_{B i}+\beta_{D i} E_{D i}^{T} E_{D i}\right)$,

$\Gamma_{i 54}=h\left(\beta_{B i} E_{B i}^{T} E_{B i}+\beta_{D i} E_{D i}^{T} E_{D i}\right)$,

$\Gamma_{i 55}=\beta_{B i} E_{B i}^{T} E_{B i}+\beta_{D i} E_{D i}^{T} E_{D i}$. 
Moreover, by Lemma 2, (15) gives

$$
\mathcal{L} V_{2}\left(x_{t}, i, t\right) \leq z_{i 2}^{T}(t) h Q z_{i 2}(t)-z_{i 4}^{T}(t) h Q z_{i 4}(t),
$$

while (16) gives

$$
\begin{aligned}
\mathcal{L} V_{3}\left(x_{t}, i, t\right)= & h z_{i 3}^{T}(t) R z_{i 3}(t) \\
& -\int_{t-h}^{t} g^{T}(r(s), s) R g(r(s), s) \mathrm{d} s .
\end{aligned}
$$

By isometry property, we have

$$
\mathbb{E}\left[z_{i 5}^{T}(t) R z_{i 5}(t)\right]=\mathbb{E}\left[\int_{t-h}^{t} g^{T}(r(s), s) R g(r(s), s) \mathrm{d} s\right] .
$$

Therefore, substituting inequalities (14)-(23) into (13) and taking expectation on the both sides yield

$$
\mathbb{E} \mathcal{L} V\left(x_{t}, i, t\right) \leq \mathbb{E}\left[z_{i}^{T}(t) \tilde{\Gamma}_{i} z_{i}(t)\right],
$$

where $\tilde{\Gamma}_{i}=\Gamma_{i}+\operatorname{diag}\{0, h Q, h R,-h Q,-R\}$.

By Schur complement, inequality (6) implies that $\tilde{\Gamma}_{i}<0$. So we have

$$
\mathbb{E} \mathcal{L} V\left(x_{t}, i, t\right) \leq-\lambda_{i 0} \mathbb{E}\left|z_{i}(t)\right|^{2} \leq-\lambda_{0} \mathbb{E}|x(t)|^{2},
$$

where $\lambda_{i 0}=\lambda_{m}\left(-\tilde{\Gamma}_{i}\right)>0$ and $\lambda_{0}=\min _{1 \leq i \leq N} \lambda_{i 0}>0$. By definition of (11), we see

$$
\alpha_{0}|x(t)|^{2} \leq V\left(x_{t}, i, t\right) \leq \alpha_{1}|x(t)|^{2}+\alpha_{2} \int_{t-2 h}^{t}|x(s)|^{2} \mathrm{~d} s
$$

for all $t \geq h$, where $\alpha_{0}=\min _{1<i<N} \lambda_{m}\left(P_{i 11}\right), \alpha_{1}=$ $\max _{1 \leq i \leq N} \lambda_{M}\left(P_{i 11}\right)$ and $\alpha_{2}=h \bar{C}_{L}^{2}\left(\lambda_{M}(Q)+\lambda_{M}(R)\right)$. Choose $\varepsilon>0$ such that

$$
\varepsilon\left(\alpha_{1}+2 h \alpha_{2} e^{2 h \varepsilon}\right) \leq \lambda_{0} .
$$

Substituting these into (12), we obtain that

$$
\begin{aligned}
& \mathbb{E}\left[e^{\varepsilon \tau} V\left(x_{\tau}, r(\tau), \tau\right)\right]-\mathbb{E}\left[e^{\varepsilon h} V\left(x_{h}, r(h), h\right)\right] \\
& =\int_{h}^{\tau} e^{\varepsilon t} \mathbb{E}\left[\varepsilon V\left(x_{t}, r(t), t\right)+\mathcal{L} V\left(x_{t}, r(t), t\right)\right] \mathrm{d} t \\
& \leq \int_{h}^{\tau} e^{\varepsilon t}\left\{\mathbb{E}\left[\varepsilon \alpha_{1}|x(t)|^{2}+\varepsilon \alpha_{2} \int_{t-2 h}^{t}|x(v)|^{2} \mathrm{~d} v\right]\right. \\
& \left.-\lambda_{0} \mathbb{E}|x(t)|^{2}\right\} \mathrm{d} t .
\end{aligned}
$$

Since

$$
\begin{gathered}
\int_{h}^{\tau} e^{\varepsilon t} \mathrm{~d} t \int_{t-2 h}^{t}|x(v)|^{2} \mathrm{~d} v \leq 2 h e^{2 h \varepsilon} \int_{-h}^{\tau}|x(t)|^{2} e^{\varepsilon t} \mathrm{~d} t \\
\leq 2 h e^{2 h \varepsilon} \int_{h}^{\tau}|x(t)|^{2} e^{\varepsilon t} \mathrm{~d} t+2 h e^{2 h \varepsilon} \int_{-h}^{h}|x(t)|^{2} \mathrm{~d} t,
\end{gathered}
$$

it follows

$$
\begin{aligned}
& e^{\varepsilon \tau} \mathbb{E} V\left(x_{\tau}, r(\tau), \tau\right) \leq e^{\varepsilon h} \mathbb{E} V\left(x_{h}, r(h), h\right) \\
& +\int_{h}^{\tau} e^{\varepsilon t}\left[\varepsilon\left(\alpha_{1}+2 h \alpha_{2} e^{2 h \varepsilon}\right)-\lambda_{0}\right] \mathbb{E}|x(t)|^{2} \mathrm{~d} t \\
& +2 h \varepsilon \alpha_{2} e^{2 h \varepsilon} \int_{-h}^{h} \mathbb{E}|x(t)|^{2} \mathrm{~d} t \leq C_{h},
\end{aligned}
$$

where $\quad C_{h} \quad=\quad e^{\varepsilon h}\left[\alpha_{1} \mathbb{E}|x(h)|^{2}+\alpha_{2}(1 \quad+\right.$ $\left.\left.2 h \varepsilon e^{h \varepsilon}\right) \int_{-h}^{h} \mathbb{E}|x(s)|^{2} \mathrm{~d} s\right]$ is a finite positive number since $x(t)$ is a unique continuous solution to equation (1). So we have

$$
\alpha_{0} e^{\varepsilon \tau} \mathbb{E}|x(\tau)|^{2} \leq e^{\varepsilon \tau} \mathbb{E} V\left(x_{\tau}, r(\tau), \tau\right) \leq C_{h} .
$$

This implies

$$
\limsup _{\tau \rightarrow \infty} \frac{1}{\tau} \log \mathbb{E}|x(\tau)|^{2} \leq-\varepsilon
$$

which completes the proof.

\section{Examples}

In this section, we present some examples to illustrate the effectiveness of the method proposed in this paper.

Example 1. Let $r(t), t>0$, be a right-continuous Markov chain taking values in $S=\{1,2\}$ with the generator

$$
\gamma=\left(\gamma_{i j}\right)_{2 \times 2}=\left[\begin{array}{cc}
-1 & 1 \\
5 & -5
\end{array}\right]
$$

Consider a scalar stochastic delay system with Markovian switching

$$
\mathrm{d} x(t)=[a(r(t)) x(t)+b(r(t)) x(t-h)] \mathrm{d} t+\sigma(r(t)) x(t-h) \mathrm{d} w(t),
$$

where $a_{1}=-1, a_{2}=1, b_{1}=b_{2}=-1$ and $\sigma_{1}=\sigma_{2}=0.1$. By the results of [5], system (32) are exponentially stable and asymptotically stable in mean square if the upper bound of time delay $h_{\max }<0.3996$ and $h_{\max }<0.4127$ respectively. However, by Theorem 1, system (32) is exponentially stable when $h \leq 0.6411$, which is much less conservative.

Example 2. Let $r(t), t>0$, be a right-continuous Markov chain taking values in $S=\{1,2\}$ with the generator

$$
\gamma=\left(\gamma_{i j}\right)_{2 \times 2}=\left[\begin{array}{cc}
-6 & 6 \\
1 & -1
\end{array}\right]
$$

Consider a scalar stochastic delay system with Markovian switching

$$
\mathrm{d} x(t)=\left(B_{r}+\Delta B_{r}\right) x(t-h) \mathrm{d} t+\Delta D_{r} x(t-h) \mathrm{d} w(t),
$$

where $B_{1}=1, B_{2}=-4, \Delta B_{i}(t) \in[-\delta, \delta]$ and $\Delta D_{i}(t) \in$ $[-\delta, \delta]$ with $\delta>0$ for $i=1,2$.

The problem of stability of system (33) was studied in Example 9.8, p342, [9]. For the case of $\delta=0.1$, inequality (9.51), p343, [9] gives $h_{\max }<0.0235$ for exponential stability while applying Theorem 1 yields $h_{\max }=0.2375$. But for the case when

$$
\delta=0.26>\frac{1}{3+0.5 \sqrt{3}}=0.2587
$$

the result in [9] does not work while Theorem 1 gives $h_{\max }=0.2175$ for exponential stability. 


\section{Conclusion}

This paper has presented a delay-dependent criterion for robust exponential stability of stochastic delay systems with Markovian switching in terms of LMIs. It should be pointed out that introduction of (9), (10) and (17) helps exploit the structure of diffusion of the system and deal with the crossing terms. This leads to a less conservative result. The above numerical examples have verified the effectiveness of the proposed method.

\section{Acknowledgment}

The authors gratefully acknowledge reviewers' comments.

\section{References}

[1] K. Gu, An integral inequality in the stability problem of time-delay systems, [C]// Proceedings of the 39th IEEE Conference on Decision and Control. Sydney: IEEE Press, 2000: 2805-2810.

[2] Y. Ji, H.J. Chizeck, Controllability, stabilizability and continuoustime Markovian jump linear quadratic control[J]. IEEE Trans. Automatic Control, 1990, 35: 777-788.

[3] X. Mao, Stochastic differential equations and applications[M]. Chichester: Horwood Publishing, 1997.

[4] X. Mao, Stability of stochastic differential equations with Markovian switching[J]. Stochastic Processes and their Applications, 1999, 79 45-67.

[5] X. Mao, L. Shaikhet, Delay-dependent stability criteria for stochastic differential delay equations with Markovian switching [J]. SACTA, 2000, 3: 87-101.

[6] X. Mao, A. Matasov and A.B. Piunovskiy, Stochastic differential delay equations with Markovian switching[J]. Bernoulli, 2000, 6: 7390.

[7] X. Mao, Exponential stability of stochastic delay interval systems with Markovian switching[J]. IEEE Trans. Automatic Control, 2002,
47: 1604-1612.

[8] X. Mao, J. Lam, S. Xu and H. Gao, Razumikhin method and exponential stability of hybrid stochastic delay interval systems[J]. J. Math. Anal. and Appl., 2006, 314: 45-66.

[9] X. Mao, C. Yuan, Stochastic differential equations with Markovian switching[M]. London: Imperial College Press, 2006.

[10] V. N. Phat, Robust stability and stabilizability of uncertain linear hybrid systems with state delays[J]. IEEE Trans. Circuits and Systems-II: Express Briefs, 2005, 52: 94-98.

[11] X.-M. Sun, W. Wang, G.-P. Liu, J. Zhao, Stability analysis for linear switched systems with time-varying delay[J]. IEEE Trans. Systems, Man, and Cybernetics tPart B: Cybernetics, 2008, 38: 528-533.

[12] Z. Wang, J. Lam, X. Liu, Exponential filtering for uncertain Markovian jump time-delay systems with nonlinear disturbances [J]. IEEE Trans. Circuits and Systems-II: Express Briefs, 2006, 53: 262268.

[13] B. Xu, Stability robustness bounds for linear systems with multiple time-varying delayed perturbations[J]. International J. Systems Science, 1997, 28: 1311-1317.

[14] S. Xu, J. Lam, X. Mao, Delay-Dependent $H_{\infty}$ Control and Filtering for Uncertain Markovian Jump Systems With Time-Varying Delays[J]. IEEE Trans. Circuits and Systems-I: Regular Papers, 2007, 54: 2070-2077.

[15] S. Yang, X. Zhengrong, Comments on "Robust stability and stabilizability of uncertain linear hybrid systems with state Delay"'[J]. IEEE Trans. Circuits and Systems-II: Express Briefs, 2006, 53: 590591.

[16] C. Yuan, $\mathrm{X}$. Mao, Robust stability and controllability of stochstic differential delay equations with Markovian switching[J]. Automatica , 2004, 40: 343-354.

[17] D. Yue, Q.-L. Han, Delay-dependent exponential stability of stochastic systems with time-varying delay, nonlinearity, and Markovian switching[J]. IEEE Trans. Automatic Control, 2005, 50: 217-222. 